\title{
Neurocognitive Workload Assessment Using the Virtual Reality Cognitive Performance Assessment Test
}

\author{
Thomas D. Parsons, Louise Cosand, Christopher Courtney, \\ Arvind Iyer, and Albert A. Rizzo \\ University of Southern California's Institute for Creative Technologies \\ Marina del Rey, CA, USA \\ \{tparsons, cosand, courtney, aiyer, arizzo\} @ict.usc.edu
}

\begin{abstract}
The traditional approach to assessing neurocognitive performance makes use of paper and pencil neuropsychological assessments. This received approach has been criticized as limited in the area of ecological validity. While virtual reality environments provide increased ecological validity, they are often done without taking seriously the demands of rigorous research design and control for potentially confounding variables. The newly developed Virtual Reality Cognitive Performance Assessment Test (VRCPAT) focuses upon enhanced ecological validity using virtual environment scenarios to assess neurocognitive processing. After an assessment for potential confounds (i.e. appropriate level of immersion and performance on neuropsychological measures), the VRCPAT battery's Attention Module (i.e. Humvee scenario) was administered to a sample of healthy adults. Findings suggest that increase in stimulus complexity and stimulus intensity can manipulate attention performance within the Attention Module.
\end{abstract}

Keywords: Neuropsychological assessment, neurocognitive, ecological validity, virtual environment.

\section{Introduction}

Attentional processing is an area of particular significance for neuropsychological research into the pattern of neurocognitve strengths and weaknesses in both normal and clinical populations. Two predominant attentional networks have emerged from studies using techniques drawn from clinical [1] and experimental neuropsychology [2], [3]. First, there is the "posterior" system which is believed to include midbrain structures and posterior parietal areas. This "posterior" system is conceptualized as being a largely bottom-up network driven by environmental salience. A second system is known as the "anterior" system which is believed to include frontal and parietal regions as well as the reticular nucleus of the brainstem. This "anterior" system is conceptualized as being a top-down regulatory network involving neurocognitively driven response control. From an applied neuropsychological perspective, this means that the "anterior" system focuses upon the voluntary maintenance of vigilance and sustained attention [4]. 
In general, findings from research related to attention may be understood in terms of automatic and controlled processing [5]. Whilst automatic processing is considered as parallel, requiring little effort, and not under the participant's direct control, controlled processing is understood as serial, effortful, and under a participant's direct control [6]. Given the effortful nature of controlled processing, it has been found to have an attentional decrement, in which reaction times slow and error rates increase as an effect of time-on-task. The distinction between automatic and controlled processing can be further defined as exogenous and endogenous attention. While exogenous attention refers to the impact of external physical events upon automatic attention, endogenous attention refers to one's active direction of attention to something deemed important by the participant [7]. Adjustments to stimulus complexity are used to assess these differing aspects of attentional processing. For example, automatic processing and endogenous attention may be assessed by having a subject stare at a computer screen that has four-digit numbers consistently presented in a fixed central location on a computer screen. Contrariwise, an example of controlled processing and exogenous attention is reflected in a scenario in which the four-digit numbers appear randomly throughout the computer screen.

Neuropsychological studies of attention tend to assess neurocognitive (e.g. neuropsychological assessment in a controlled setting) and behavioral (e.g. self and other behavioral rating scales of the subject's activities in a real-world setting) aspects of attention. It is important to note that neurocognitive measures in controlled settings and behavioral ratings based upon naturalistic observations do not consistently proffer parallel findings [8]. Further, dissimilar attentional components may be dissociated both by neurocognitive measures in controlled settings and behavioral ratings based upon naturalistic observations [3]. A related issue is that while traditional neuropsychological assessments manipulate the complexity of the stimulation, they do little to assess the impact of the intensity of the situation. The assessment of attention should reflect the varying levels of intensity found in real world situations. A more intense setting may elicit emotional responses. Findings from attentional assessments must be generalizable to real-world situations [9]. While controlled settings offer increased psychometric rigor, naturalistic observation-based behavioral ratings may better capture the subject's performance in a real world setting.

\subsection{Virtual Environments for Neuropsychological Assessment}

Virtual Reality offers the capacity for merging the benefits of controlled settings (e.g. increased psychometric rigor) within environments that simulate the environment in which naturalistic observation-based behaviors occur. Recent advances in simulation technology have produced new methods for the creation of virtual environments. With these systems, users can proffer ecological verisimilude reflective of "real world" environments. When delivered via an immersive head-mounted display (HMD), an experience of presence within these captured scenarios can be supported in human users. As such, the VR assets that allow for precise stimulus delivery within ecologically enhanced scenarios appears well matched for research into attentional processing.

The value in using virtual reality technology to produce simulations targeting neurocognitive and behavioral applications has been acknowledged by an 
encouraging body of research. Some of the work in this area has addressed affective processes: anxiety disorders, pain distraction, posttraumatic stress disorder [10]. Other work has assessed neurocognitive processes such as attention and executive functioning [11], [12]; memory [13], [14], [15]; and visuospatial abilities [16], [17], [18]. While multiple attempts have been made to apply theoretical perspectives to the development of believable virtual environments, little has been done to "objectively" assess human interpretations of these environments. There is need for the incorporation of psychophysiological metrics into assessment of persons responses while in a virtual environment. As mentioned above, attentional assessment should aim to recreate the environment in which the subject will be processing information. This is especially important when persons are processing information in environments that have different levels of stimulus intensity. Exposure to emotionally intense situations results in regular activation of cerebral metabolism in brain areas associated with inhibition of maladaptive associative processes [19]. Identical neural circuits have been found to be involved in affective regulation across affective disorders [20], [21]. Systematic and controlled exposure to physiologically intense stimuli may enhance emotional regulation through adjustments of inhibitory processes on the amygdala by the medial prefrontal cortex during exposure and through structural changes in the hippocampus [22].

Thus far, the recording of psychophysiological variables while participants operate within virtual environments has produced useful results in studies examining attention and presence [23], [24], [25]. As such, the VR assets that allow for precise stimulus delivery within ecologically enhanced scenarios appears well matched for this research. Researchers have found that the individual characteristics of study participants may impact the immersiveness and subsequent findings of a given study. Of primary importance is the extent to which a participant is capable of "absorption" and "hypnotism." Hence, individual differences may moderate presence and confound findings. The propensity of participants to get involved passively in some activity and their ability to concentrate and block out distraction are important factors to consider when conducting a study. Likewise, evidence suggests that hypnotizability plays a role in the outcome of studies using VR. Research into these moderating individual traits is of value because such research may augment participant selection.

\subsection{Virtual Reality Cognitive Performance Assessment Test}

The project described herein builds upon a larger (ongoing) project that makes use of virtual environments to assess user sensory, perceptual, and neurocognitive performance on various tasks. Neurocognitive and psychophysiological data gleaned from such analyses provides opportunity for implementing systems that can exploit the capabilities of nervous systems, rather than simply depending upon human adaptation, to improve and optimize human-computer interaction. Monitoring the neurocognitive and psychophysiological activity of persons operating within a complex environment, however, poses severe measurement challenges. It is also likely that neurocognitive and psychophysiological responses in operational versus tightly controlled laboratory environments will be significantly, if not fundamentally, different than in controlled laboratory settings. 
The Virtual Reality Cognitive Performance Assessment Test (VRCPAT) project focuses on the refinement of neuropsychological assessment using virtual environments to assess persons immersed in ecologically valid virtual scenarios. The VRCPAT is a three-dimensional virtual environment (i.e. virtual city and Humvee scenarios) designed to run on a Pentium IV notebook computer with one gigabyte RAM and a 128 megabyte graphics card. The primary aim of the VRCPAT project is to use the already existing library of assets as the basis for creating a VE for the standardized assessment of neurocognitive performance within a contextually relevant VE. The application uses USC's FlatWorld Simulation Control Architecture (FSCA). The FSCA enables a network-centric system of client displays driven by a single controller application. The controller application broadcasts user-triggered or scripted-event data to the display client. The real-time three-dimensional scenes are presented using Numerical Design Limited's (NDL's) Gamebryo graphics engine. The content was edited and exported to the engine, using Alias's Maya software. Three-dimensional visual imagery is presented using the eMagin z800. Navigation through the scenario uses a common USB Logitech game pad device.

Virtual reality-based simulation technology approaches, as delineated herein, are considered to be the future alternative for devising neuropsychological assessment measures that will have better ecological/predictive validity for real-world performance. As well, the flexibility of stimulus delivery and response capture that are fundamental characteristics of such digital environments is viewed as a way for research objectives to be addressed in a more efficient fashion for long term needs. The overall design of this type of assessment tool allows for 1) Verisimilitude: the presentation of realistic environments that reflect activities of daily living; and 2) Veridicality: flexibility in terms of the independent variables that could be studied with this method once the psychometric properties of the standardized test are determined. Such flexibility enables this system to be viewed as an open platform on which a wide range of research questions may be addressed. These include the manipulation of: 1) information load on the front end via the intensity and complexity of target stimuli to be attended to and the type of information in terms of relevance, similarity, vagueness, sensory properties; 2) temporal constraints during varied sustained assessment conditions; 3) distracting activities during the neurocognitive assessments; 4) sensory modality of the information presentation that needs to be attended to; 5) the reward structure used during some tests to assess motivational factors that influence performance; 6) the presentation of aversive stimuli for stressed performance evaluations; and 7) the development of a test bed whereby neurocognitive training and augmented cognition strategies could be assessed under known conditions supported by normative standards.

\section{Methods}

\subsection{Participants}

The study sample initially included 21 healthy adults made up of 15 civilians (i.e. USC students); and 6 military subjects (i.e. West Point Cadets). After an analysis of the impact of immersion as a potential confound upon attentional assessment, the 
military cohort was removed (see below). The resulting subject pool included 15 healthy subjects $($ Age, mean $=26.71, \mathrm{SD}=4.49 ; 50 \%$ male; and Education, mean $=$ 15.50 , SD = 2.54). Strict exclusion criteria were enforced so as to minimize the possible confounding effects of additional factors known to adversely impact cognition, including psychiatric (e.g., mental retardation, psychotic disorders, diagnosed learning disabilities, Attention-Deficit/Hyperactivity Disorder, and Bipolar Disorders, as well as substance-related disorders within two years of evaluation) and neurologic (e.g., seizure disorders, closed head injuries with loss of consciousness greater than 15 minutes, and neoplastic diseases) conditions. Subjects were comparable in age, education, ethnicity, sex, and self-reported symptoms of depression.

\subsection{Procedure}

The University of Southern California's Institutional Review Board approved the study. Experimental sessions took place over a two hour period. After informed consent was obtained, basic demographic information and computer experience and usage activities were recorded. Subjects then completed a neuropsychological battery administered under standard conditions. Following completion of the neuropsychological battery, subjects completed the simulator sickness questionnaire, which includes a pre-VR exposure symptom checklist. Next, all participants were administered the VRCPAT as part of a larger neuropsychological test battery. While experiencing the VRCPAT, participant psychophysiological responses were reorded using the Biopac system.

\subsection{Potential Confounds: Immersion and Neuropsychological Assessment}

The impact of highly immersive VR on participants' psychophysiological responses was compared with responses to a less immersive experience of watching the scenario on a laptop screen. The "high immersion" condition utilized a head-mounted display, headphones, and a tactile transducer. In the "low immersion" condition, participants wore headphones and watched the scene on a laptop computer screen. The stimuli included a virtual environment, in which the participants experienced "high intensity" and "low intensity" scenarios that occurred while participants drove a Humvee. Stimulus "intensity" was modulated by placing the user in "safe" (low intensity) and "ambush" (high intensity) settings: start section; palm ambush; safe zone; city ambush; safe zone; and bridge ambush. While participants drove the Humvee through the virtual environment scenarios, they were intermittently probed with acoustic startles (110 dB acoustic startle probes). Measures included two psychophysiological measures (startle eyeblink amplitude and heart rate) and responses on two self-report questionnaires (Tellegen Absorption Scale and Immersive Tendencies Questionnaire).

The following paper and pencil neuropsychological measures were used to asses for potential confounding differences within the subject pool: To assess Attention we used Digit Span (Forward and Backward) from the Wechsler Adult Intelligence Scale-Third edition (WAIS-III). To assess processing speed we used Digit Symbol Coding from the WAIS-III, and Trail Making Test Part A (TMT). To assess executive functioning we used TMT Part B; and the Stroop Color and Word Test. To assess verbal learning and memory we used the Hopkins Verbal Learning Test - Revised 
(HVLT-R); to assess nonverbal learning and memory we used the Brief Visuospatial Memory Test - Revised (BVMT-R); and to assess Lexical-Semantic Memory we used Controlled Oral Word Association Test (FAS); 2) Semantic Fluency (Animals).

\subsection{VRCPAT Humvee Attention Module}

The VRCPAT portion included a HUMVEE Attention Task. The Humvee scenario assessed attention using varying levels of both stimulus "intensity" and stimulus "complexity". Manipulation of stimulus intensity included low intensity situations "safe zones" and high intensity situations "ambush zones": 1) start section; 2) palm ambush; 3) safe zone; 4) city ambush; 5) safe zone; 6) bridge ambush. The manipulation of stimulus complexity involved the presentation of a four-digit number that was superimposed on the virtual windshield (of the Humvee) while the subject drove the Humvee. Each four-digit number was presented for approximately $300 \mathrm{~ms}$ and was randomly selected by the computer from a database of prescreened numbers. During low (simple) complexity presentations the numbers were continually presented in a fixed central location on the windshield. During high complexity presentations the numbers were presented randomly throughout the windshield.

The design consisted of six Humvee attention conditions:

1. Fixed Position: 2.0 second condition (Start Section): In this condition, the fourdigit number always appeared in a fixed central location on the "windshield." The numbers were presented at 2.0 second intervals. This occurred in the "Start Section" and ended just before the "Palm Ambush."

2. Fixed Position: 1.5 second condition (Palm Ambush): The procedure for this condition was identical to the "Fixed Position" condition described previously except that the numbers were presented at 1.5 second intervals. This occurred in the "Palm Ambush" section and ended just before the "Safe Zone" section.

3. Fixed Position: 0.725 second condition (Safe Zone): The procedure for this condition was identical to the "Fixed Position" condition described previously except that the numbers were presented at 0.725 second intervals. This occurred in the "Safe zone" and ended just before the "City Ambush" section.

4. Random Position: 2.0 second condition (City Ambush): The procedure for this condition is similar to the "Fixed Position" condition with the exception that the numbers appear randomly throughout the "windshield" rather than in one fixed central location. The numbers were presented at 2.0 second intervals. This occurred in the "City Ambush" and ended just before the "Safe Zone".

5. Random Position: 1.5 second condition (Safe Zone): The procedure for this condition is similar to the preceding "Random Position" condition except that the numbers were presented at 1.5 second intervals. This occurred in the "Safe Zone" and ended just before the "Bridge Ambush".

6. Random Position: 0.725 second condition (Bridge Ambush): The procedure for this condition is similar to the preceding "Random Position" condition except that the numbers were presented at 0.725 second intervals. This occurred in the "Bridge Ambush". 


\section{Results}

To examine potential cohort confounds, the impact of high versus low levels of immersive virtual reality on participants' $(\mathrm{N}=14$ : six West Point cadets and eight University of Southern California student civilians) psychophysiological responses was compared.

\subsection{Assessment of Potential Confounds}

Given the similarity of participants in terms of age, sex, education, ethnicity, immersiveness, and performance on standard paper and pencil measures of neuropsychological assessments, no correction for these variables was employed. Notably, none of the participants reported simulator sickness following VR exposure as measured by the SSQ. West Point cadets, however, responded with significantly lower eyeblink amplitudes $(\mathrm{F}=7.249$, $\mathrm{p}<0.05)$ overall. Participants in the "high immersion" condition, cadet or civilian, had higher eyeblink amplitudes than did the participants in the "low immersion" condition. A significant interaction between condition ("high immersion" condition and "low immersion" condition) and participant group (West Point cadets and University of Southern California students) was also found in relation to heart rate. West Point cadets had significantly slower heart rates during the "low immersion" condition compared to University of Southern California students $(\mathrm{F}=17.662, \mathrm{p}<0.001)$, while their average median heart rates in the "high immersion" condition were nearly identical (West Point cadet mean $=0.802$, University of Southern California student mean $=0.799, \mathrm{~F}=0.001, \mathrm{p}=0.997$ ).

\subsection{Analyses after Controlling for Confounded Data}

As a result of the above findings a secondary analysis was done in which the West Point cadets were excluded. Given the similarity of participants in the civilian cohort in terms of age, sex, education, ethnicity, immersiveness, and performance on standard paper and pencil measures of neuropsychological assessments, no correction for these variables was employed. Again, none of the participants reported simulator sickness following VR exposure as measured by the SSQ.

Analyses of Immersion Level's Impact upon Users. To examine differences in levels of immersion upon this new cohort, one-way ANOVAs were performed, comparing median startle eyeblink amplitudes in "high immersion" (Mean = 0.29; SD $=0.09$ ) versus "low immersion" scenarios (Mean $=0.18 ; \mathrm{SD}=0.03$ ). The results indicated that the increase in immersion caused a significant increase in median startle eyeblink amplitudes $(\mathrm{F}=19.17$; $\mathrm{p}<0.001)$. Participants' cardiac responses showed a similar trend as the median beats per minute (BPM) in the "high immersion" condition (Mean $=86.71$; $\mathrm{SD}=47.75$ ) were higher than median BPM in the "low immersion" condition (Mean $=61.21 ; \mathrm{SD}=11.29$ ). This trend approached significance $(\mathrm{F}=7.918 ; \mathrm{p}<0.005)$, corroborating the EMG finding that "high immersion" scenarios evoke a stronger physiological reaction than "low immersion" scenarios. 
Analyses of Attentional Processing. To examine scenario differences related to the "complexity" of stimulus presentation, one-way ANOVAs were performed, comparing attentional performance in "simple" stimulus presentations (Mean = 43.63; $\mathrm{SD}=8.91$ ) versus "complex" stimulus presentations (Mean $=34.63 ; \mathrm{SD}=6.86$ ). The results indicated that the increase in stimulus complexity caused a significant decrease in performance on attentional tasks $(\mathrm{F}=5.12 ; \mathrm{p}=0.04)$.

To examine scenario differences related to the "intensity" of stimulus presentation, we compared attentional performance in low intensity (Mean $=40.01 ; \mathrm{SD}=4.06$ ) versus high intensity (Mean $=9.25 ; \mathrm{SD}=3.70)$ presentations. The results indicated that the increase in stimulus intensity caused a significant decrease in performance on attentional tasks $(\mathrm{t}=9.83 ; \mathrm{p}=0.01)$.

It is important to note that a confound was not found in the distribution of the standard neuropsychological assessment scores. Given the small sample size, we decided to not assess the construct validity of the VRCPAT Attention Modules. Hence, no attempts were made to assess correlations between standard paper and pencil tests and VRCPAT.

\section{Conclusions}

Our goal was to conduct an initial pilot study of the general usability of the VRCPAT Attention Module scenarios. We aimed at assessing whether the increase in stimulus complexity would result in a significant decrease in performance on attentional tasks. We also wanted to see whether an increase in stimulus intensity would result in a significant decrease in performance on attentional tasks. We believe that this goal was met as the study results indicated that: (1) the increase in stimulus complexity caused a significant decrease in performance on attentional tasks; and 2) the increase in stimulus intensity caused a significant decrease in performance on attentional tasks.

We also aimed to assess the impact of potential confounds (cohort, immersion, and performance on traditional neuropsychological assessments) upon neurocognitive performance within virtual environments. First, a cohort confound was found. Results suggest that although West Point cadets found the virtual environment to be a less negative experience than did the University of Southern California student controls, the "high immersion" condition was a more emotionally salient condition. Hence, highly immersive VEs may be effective training tools in simulating military scenarios. It is important to note that a confound was not found in the distribution of the standard neuropsychological assessment scores. This is important and may reflect construct validation. However, this must be corroborated with an increased sample size and a multitrait-multimethod matrix analysis, in which convergent and discriminant validity would be assessed.

Our findings should be understood in the context of some limitations. First, these findings are based on a small sample size. As a necessary next step, the reliability and validity of the test needs to be established using a larger sample of participants. This will ensure that the current findings are not an anomaly due to sample size. Additionally, the diagnostic utility of this attention assessment tool must be determined. The ability of the VRCPAT's Attention Module to accurately classify participants into attention impaired and attention intact groups based on carefully 
established critical values must be evaluated. This will involve the generation of specific cut-off points for classifying a positive or negative finding. The VRCPAT Attention Module's prediction of attentional deficits will need to be evaluated by the performance indices of sensitivity, specificity, predictive value of a positive test, and predictive value of a negative test.

In sum, manipulation of stimulus complexity and intensity in the VRCPAT's Attention Module revealed significant differences in performance on attentional tasks. Complementary comparisons of the VRCPAT's Attention Module with standardized behavioral and neurocognitive tests developed to assess attentional abilities are also warranted in an increased sample size to determine the VRCPAT's construct validity.

\section{References}

1. Mirsky, A.F., Anthony, B.J., Duncan, C.C., Ahearn, M.B., Kellam, S.G.: Analysis of the elements of attention: A neuropsychological approach. Neuropsychology Review 2, 109145 (1991)

2. Posner, M.I., Petersen, S.E.: The attention system of the human brain. Annual Review of Neuroscience 13, 25-42 (1990)

3. Knudsen, E.I.: Fundamental components of attention. Annual Review of Neuroscience 30, 57-78 (2007)

4. Posner, M.I., Raichle, M.E.: Networks of attention. In: Posner, M.I., Raichle, M.E. (eds.) Images of mind, pp. 153-179. Scientific American, New York (1994)

5. Fisk, A.D., Schneider, W.: Control and automatic processing during tasks requiring sustained attention: a new approach to vigilance. Hum Factors 23, 737-750 (1981)

6. Schneider, W., Shiffrin, R.M.: Controlled and automatic human information-processing. 1. Detection, search, and attention. Psychol. Rev. 84, 1-66 (1977)

7. Posner, M.I.: Orienting of attention. Q. J. Exp. Psychol. 32, 3-25 (1980)

8. Gordon, M., Barkley, R.A., Lovett, B.J.: Tests and observational measures. In: Barkley, R.A. (ed.) Attention-deficit hyperactivity disorder: A handbook for diagnosis and treatment, 3rd edn., pp. 369-388. Guilford, New York (2006)

9. Foa, E.B., Kozak, M.J.: Emotional processing of fear: exposure to corrective information. Psychological Bulletin 99, 20-35 (1986)

10. Parsons, T.D., Rizzo, A.A.: Affective Outcomes of Virtual Reality Exposure Therapy for Anxiety and Specific Phobias: A Meta-Analysis. Journal of Behavior Therapy and Experimental Psychiatry 39, 250-261 (2008)

11. Parsons, T.D., Bowerly, T., Buckwalter, J.G., Rizzo, A.A.: A controlled clinical comparison of attention performance in children with ADHD in a virtual reality classroom compared to standard neuropsychological methods. Child Neuropsychology 13, 363-381 (2007)

12. Parsons, T.D., Rizzo, A.A.: Neuropsychological Assessment of Attentional Processing using Virtual Reality. Annual Review of CyberTherapy and Telemedicine 6, 23-28 (2008)

13. Parsons, T.D., Rizzo, A.A., Bamattre, J., Brennan, J.: Virtual Reality Cognitive Performance Assessment Test. Annual Review of CyberTherapy and Telemedicine 5, 163-171 (2007)

14. Parsons, T.D., Rizzo, A.A.: Initial Validation of a Virtual Environment for Assessment of Memory Functioning: Virtual Reality Cognitive Performance Assessment Test. Cyberpsychology and Behavior 11, 17-25 (2008) 
15. Parsons, T.D., Silva, T.M., Pair, J., Rizzo, A.A.: A Virtual Environment for Assessment of Neurocognitive Functioning: Virtual Reality Cognitive Performance Assessment Test. Studies in Health Technology and Informatics 132, 351-356 (2008)

16. Parsons, T.D., Larson, P., Kratz, K., Thiebaux, M., Bluestein, B., Buckwalter, J.G., Rizzo, A.A.: Sex differences in mental rotation and spatial rotation in a virtual environment. Neuropsychologia 42, 555-562 (2004)

17. Parsons, T.D., Rizzo, A.A., Buckwalter, J.G.: Backpropagation and regression: comparative utility for neuropsychologists. Journal of Clinical and Experimental Neuropsychology 26, 95-104 (2004)

18. Parsons, T.D., Rizzo, A.A., van der Zaag, C., McGee, J.S., Buckwalter, J.G.: Gender and cognitive performance: a test of the common cause hypothesis. Aging, Neuropsychology, and Cognition 12, 78-88 (2005)

19. Schwartz, J.M.: Neuroanatomical aspects of cognitive-behavioural therapy response in obsessivecompulsive disorder. An evolving perspective on brain and behaviour. British Journal of Psychiatry Supplemental, 38-44 (1998)

20. De Raedt, R.: Does neuroscience hold promise for the further development of behavior therapy? The case of emotional change after exposure in anxiety and depression. Scandinavian Journal of Psychology 47, 225-236 (2006)

21. Mineka, S., Watson, D., Clark, L.A.: Comorbidity of anxiety and unipolar mood disorders. Annual Review of Psychology 49, 377-412 (1998)

22. Hariri, A.R., Bookheimer, S.Y., Mazziotta, J.C.: Modulating emotional responses: effects of a neocortical network on the limbic system. Neuroreport 11, 43-48 (2000)

23. Macedonio, M., Parsons, T.D., Rizzo, A.A.: Immersiveness and Physiological Arousal within Panoramic Video-based Virtual Reality. Cyberpsychology and Behavior 10, 508$516(2007)$

24. Meehan, M., Insko, B., Whitton, M., Brooks, F.: Physiological measures of presence in virtual environments. In: Proceedings of 4th Annual Presence Workshop, Philadelphia (May 2002)

25. Pugnetti, L., Meehan, M., Mendozzi, L.: Psychophysiological correlates of virtual reality: a review. Presence 10, 384-400 (2001) 